\title{
Semantic Web Search through Natural Language Dialogues
}

\author{
Dora Melo \\ Coimbra Business School - ISCAC, IPC, and LISP-Laboratory of Informatics, Systems and \\ Parallelism, Portugal \\ Irene Pimenta Rodrigues \\ University of Évora, and LISP - Laboratory of Informatics, Systems and Parallelism, Portugal \\ Vitor Beires Nogueira \\ University of Évora, and LISP - Laboratory of Informatics, Systems and Parallelism, Portugal
}

\begin{abstract}
The Semantic Web as a knowledge base gives to the Question Answering systems the capabilities needed to go well beyond the usual word matching in the documents and find a more accurate answer, without needing the user intervention to interpret the documents returned. In this chapter, the authors introduce a Dialogue Manager that, throughout the analysis of the question and the type of expected answer, provides accurate answers to the questions posed in Natural Language. The Dialogue Manager not only represents the semantics of the questions but also represents the structure of the discourse, including the user intentions and the questions' context, adding the ability to deal with multiple answers and providing justified answers. The system performance is evaluated by comparing with similar question answering systems. Although the test suite is of small dimension, the results obtained are very promising.
\end{abstract}

Keywords: Natural Language Processing, Ontologies, Question Answering Systems, Cooperative Question Answering, Collaborative Search, Controlled Dialogue, Dialogue Manager, Answer Extraction, Answer Processing.

\section{INTRODUCTION}

The Semantic Web (SW), presented by Tim Berners-Lee (2001), has been recognized as the next step in the evolution of the World Wide Web. The inclusion of semantic contents on web pages allows machines to process such information and enables users to find, share and combine this information more easily. Question Answering (QA) systems for Natural Language (NL) on the SW besides establishing the correspondence between words in documents, they must also find a precise answer, without user's help to interpret the documents returned - the use of knowledge and reasoning to interpret and to obtain the answers (Saint-Dizier \& Moens, 2011).

Consistent with the role of ontologies in structuring and organizing semantic information on the web, QA systems based on ontologies allow exploring the expressive power of ontologies and enriching the queries' interpretation. Ontologies and the SW (Horrocks, 2008) have become formalisms able to represent the conceptual domains of knowledge and promote the capabilities of QA systems based on semantics (Guo \& Zhang, 2009). 